\title{
Obesidade e doença renal: aspectos fisiopatológicos
}

\section{Obesity and kidney disease: pathophysiological aspects}

João Eduardo Cascelli Schelb Scalla Pereira ${ }^{1}$

Danielle Guedes Andrade Ezequiel $^{2}$,

Mônica Barros Costa² Rogério Baumgratz de Paula ${ }^{2}$

${ }^{1}$ Hospital Universitário da Universidade Federal de Juiz de Fora (HU-UFJF).

${ }^{2}$ Departamento de Clínica Médica - Faculdade de Medicina, Universidade Federal de Juiz de Fora, Juiz de Fora, MG.

\section{ه João Eduardo Pereira Rua Oscar Vidal, 147/602 CEP: 36010-060 \\ Juiz de Fora, MG \\ Ð joaoeschelb@gmail.com}

\section{RESUMO}

A epidemia de obesidade observada nas últimas décadas é acompanhada de aumento exponencial de doenças crônicas relacionadas, com destaque diabetes mellitus tipo 2, hipertensão arterial sistêmica, dislipidemia e doenças cardiovasculares. Do mesmo modo, a obesidade constitui fator de risco independente para o desenvolvimento de doença renal crônica, condição associada a elevados índices de morbidade e de mortalidade. A obesidade causa lesão renal de maneira indireta, por meio de sua estreita associação com hipertensão arterial sistêmica e com diabetes mellitus tipo 2 e de maneira direta, ao induzir adaptações glomerulares que culminam na glomerulopatia específica da obesidade. Além disso, o excesso de peso contribui para o agravamento de glomerulopatias pré-existentes. Múltiplos fatores explicam o desenvolvimento e o agravamento das lesões renais associadas à obesidade, em especial alterações hemodinâmicas, inflamatórias e metabólicas. Nesse contexto, a redução do peso corporal com ênfase nas alterações metabólicas e inflamatórias bem como o tratamento da hipertensão arterial e do diabetes mellitus constituem o primeiro passo para a prevenção primária e secundária do desenvolvimento de doença renal crônica. Nesta revisão serão apresentados os principais mecanismos fisiopatológicos da lesão renal associada à obesidade.

Palavras-chave: obesidade, doença renal crônica, glomeruloesclerose segmentar e focal, síndrome metabólica, sobrepeso

\section{ABSTRACT}

In the last decades, the obese epidemic has been accompanied by an exponential increase in the prevalence of chronic conditions such as type 2 diabetes, hypertension, dyslipidemia, and other forms of cardiovascular disease. Moreover, obesity is an independent risk factor for the development of chronic kidney disease, itself a condition associated with high risk of morbidity and mortality. Obesity can cause chronic kidney disease both indirectly, as a major risk factor for diabetes and hypertension, and directly, through adaptive glomerular changes that evolve into a specific form of glomerulopathy. Furthermore, excessive weight can worsen other forms of pre-existent glomerular diseases. Multiple factors can explain the development and worsening of renal lesions associated with obesity, mainly through hemodynamic, inflammatory and metabolic changes. In this context, reduction of body weight with emphasis on metabolic and inflammatory changes, as well as the treatment of hypertension and diabetes, constitute the first step towards primary and secondary prevention against development of chronic kidney disease. This review presents the physiopathology of the obesity related glomerulopathy.

Keywords: obesity, chronic kidney disease, focal segmental glomerulosclerosis, metabolic syndrome, overweight 


\section{INTRODUÇÃO}

A pandemia de obesidade tornou-se um problema global de saúde pública. Entre os anos 1978 e 2013, a proporção de indivíduos com sobrepeso (IMC $\geq 25 \mathrm{~kg}$ / $\mathrm{m}^{2}$ ) e obesidade (IMC $\geq 30 \mathrm{~kg} / \mathrm{m}^{2}$ ) aumentou de $28,8 \%$ para $36,9 \%$, entre os homens e de $29,8 \%$ para $38 \%$, entre as mulheres (D'AGATI et al., 2016). No Brasil, segundo o Sistema de Vigilância de Fatores de Risco e Proteção para Doenças Crônicas por Inquérito Telefônico (VIGITEL), o número de pessoas acima do peso aumentou $26,3 \%$ em 10 anos, passando de 42,6\%, em 2006 para 53,6\%, em 2016. No mesmo período, considerando apenas os pacientes obesos (IMC $\geq 30 \mathrm{~kg}$ / $\mathrm{m}^{2}$ ), o aumento foi de $60 \%$, elevando-se de $11,8 \%$ para $18,9 \%$, com frequência semelhante em ambos os sexos e com leve predomínio entre as mulheres (MINISTÉRIO DA SAÚDE, 2016). No estudo longitudinal de saúde do adulto, (ELSA), que avaliou mais de 15 mil indivíduos nas diversas capitais do Brasil, entre o período de 2009 e 2013 , observou-se que $65,9 \%$ dos homens e $60,8 \%$ das mulheres apresentavam excesso de peso (SCHIMIDT et al., 2014). O mesmo foi observado em estudo que incluiu amostra representativa da população de Juiz de Fora, no qual dentre os 1.032 indivíduos avaliados, observou-se que $61,6 \%$ se encontravam acima do peso (sobrepeso ou obesidade) (MIRANDA et al., 2017).

Em paralelo à epidemia de obesidade, observa-se aumento do número de casos de doenças crônicas a ela relacionadas. Segundo o National Health and Nutririon Examination Study III (NHANES III), que envolveu mais de 16 mil participantes, a obesidade se associou a aumento na prevalência de diabetes tipo 2 (DM2), hipertensão arterial sistêmica (HAS), doença arterial coronariana, dislipidemia, neoplasias e osteoartrose. Indivíduos obesos também são mais propensos ao desenvolvimento de doença renal crônica (DRC) ou ao agravamento de DRC preexistente. Em concordância, uma coorte histórica, mostrou que tanto o sobrepeso quanto a obesidade constituem fatores de risco independentes para o desenvolvimento de DRC. Nessa coorte, o risco relativo de DRC aumentou de 1,87 , em indivíduos com sobrepeso para 7,07, em casos de obesidade grau 3 (IMC $\geq 40 \mathrm{~kg} / \mathrm{m} 2$ ) (HSU et al., 2006).

A obesidade gera aumento da demanda metabólica corporal, que compromete o funcionamento adequado dos rins e leva à perda progressiva da função renal. Além disso, os distúrbios metabólicos presentes na obesidade contribuem de modo importante para a lesão renal, em indivíduos com excesso de peso ( $\mathrm{ZHU}$; SCHERER, 2018). Nesse sentido, o entendimento dos mecanismos fisiopatológicos responsáveis por esta associação é ferramenta importante para implantação de possíveis medidas preventivas ou terapêuticas da lesão renal em obesos.

\section{REVISÃO DE LITERATURA E DISCUSSÃO}

\section{Mecanismos de lesão renal na obesidade}

Além dos danos indiretos que a obesidade pode provocar nos rins, através da estreita associação com o DM2 e a HAS ambas consideradas as principais causas de DRC no mundo, indivíduos obesos estão expostos a danos renais diretos mediados por alterações da hemodinâmica renal além de alterações metabólicas e inflamatórias (NAUMNIK; MYSLIWIEC, 2010; SILVA JUNIOR et. al., 2017).

\section{Obesidade e hemodinâmica renal}

A obesidade induz substancial aumento da demanda metabólica, que cursa com hipertensão glomerular associada com aumento do fluxo plasmático renal, da fração de filtração, da reabsorção de sódio e do ritmo de filtração glomerular. Em estudos com cães tornados obesos, observou-se elevação do fluxo plasmático renal e da TFG, bem como balanço positivo de sódio, HAS e albuminúria (DE PAULA; SILVA; HALL, 2004). Em animais obesos, observa-se expansão da cápsula de Bowman e aumento da matriz mesangial, sendo estas alterações associadas a maior expressão de TGF $\beta 1$ (transforming growth fator beta 1) glomerular e aumento dos níveis de glicosaminoglicanos na membrana basal tubular (HENEGAR; BIGLER; HENEGAR, 2001; CHAGNAC; WEINSTEIN; KORZETS, 2000).

A hiperfiltração renal observada nos obesos é provavelmente secundária ao aumento da demanda metabólica, que eleva o débito cardíaco, a pressão arterial (PA) e o fluxo sanguíneo a órgãos não adiposos, como os rins. Este fenômeno é acompanhado de vasodilatação da arteríola aferente e hiperfiltração glomerular. Como consequência, ocorre aumento da reabsorção de sódio nos segmentos proximais do néfron, com redução da oferta desse íon aos segmentos distais e ativação da mácula densa. Uma vez ativado, esse mecanismo denominado feedback tubuloglomerular, ocorre vasodilatação aferente e secreção de renina, o que culmina em expansão do volume extracelular e vasoconstrição da arteríola eferente. Deste modo, ocorreria balanço positivo de sódio associado com maior estresse glomerular (HALL et. al., 1993; HALL, 2003; CHAGNAC; WEINSTEIN; KORZETS, 2000).

Um segundo mecanismo propõe que o evento principal seja a hiperativação do sistema reninaangiotensina (SRA) secundária ao aumento da reabsorção proximal de sódio. Uma vez ativado, - SRA promove vasoconstrição preferencial da arteríola eferente com acentuação da hipertensão intraglomerular levando ao desenvolvimento de HA resistente, hipertrofia da camada média, lesão endotelial e aumento do risco cardiovascular (D'AGATI 
et al., 2016; ZHU; SCHERER, 2018; FRIGOLET; TORRES; TOVAR, 2013). Considerando que até um terço da produção de angiotensinogênio tem origem nos adipócitos, quanto maior o grau de obesidade, maior o nível circulante dessa molécula, o que culmina com a hiperatividade do SRA (ZHU; SCHERER, 2018). Estes dados estão de acordo com modelos animais de deleção específica do angiotensinogênio do tecido adiposo, o que implica em redução significativa da PA sem, no entanto, alterar a massa do tecido adiposo (YIANNIKOURIS et al., 2012). Por outro lado, a superexpressão do angiotensinogênio específico do tecido adiposo causa aumento da PA e alterações morfológicas e funcionais renais, independente da massa de adipócitos (MASSIERA et al., 2001).

É sabido que o angiotensinogênio é convertido em sua forma metabolicamente ativa, a angiotensina II, após passar por uma série de processos enzimáticos e exerce suas funções através de seus receptores 1 e 2 (AT1 e AT2). Os receptores AT1 são acoplados à proteína $G$ e exercem a maior parte das funções do SRA, quais sejam vasoconstrição, aumento da sede, produção de aldosterona, aumento da reabsorção de sódio, ativação do sistema nervoso simpático, hipertrofia e fibrose (NGUYEN; TOUYZ, 2011). Os receptores AT2, por sua vez, desempenham funções opostas: vasodilatação, natriurese além de ações anti-inflamatória e antifibrótica (LAMARIÉ; SCHIFFRIN, 2010). Em estudos experimentais com ratos submetidos à dieta rica em gordura, observou-se aumento da expressão dos receptores AT1 nos rins e no tecido adiposo (COLE et al., 2010; GUO et al., 2017). Ainda em outros modelos animais, a deleção da isoforma dominante do AT1 não provoca HAS, mas causa danos estruturais aos rins, como expansão mesangial e vacuolização tubular (MA et al., 2011).

Por fim, de maneira complementar à maior produção de angiotensina II, indivíduos obesos apresentam aumento da secreção de fatores liberadores de mineralocorticoides provenientes dos adipócitos e desenvolvem hiperaldosteronismo secundário. Este aumento é demonstrado em modelos experimentais e humanos de obesidade, diabetes mellitus e de doenças cardiovasculares (HIRATA et al., 2012; GUO et al., 2008), e são corroborados por estudos com fármacos que bloqueiam os receptores de mineralocorticoides, como a eplerenona e espironolactona, que melhoram sobremaneira o controle pressórico e as alterações metabólicas e vasculares em indivíduos obesos. (DE PAULA; SILVA; HALL, 2004; TIROSH; GARG; ADLER, 2010). Acredita-se que a aldosterona provoque dano renal por contribuir com alterações da hemodinâmica renal, predispondo à hiperfiltração e à retenção de sódio, à semelhança do que ocorre com a angiotensina II, mas também elevando a produção de fatores de crescimento e espécies reativas de oxigênio, com consequente inibição da degradação da matriz extracelular e fibrose (REMUZZI; CATTANEO; PERICO, 2008).

Independente dos mecanismos, a hiperfiltração glomerular, associada ou não a aumento da excreção urinária de albumina e, por vezes, de proteinúria nefrótica, pode implicar em progressão de DRC. Nestes casos a lesão histológica mais frequente é representada pela glomeruloesclerose segmentar e focal (GESF). Este mecanismo, demonstrado em estudos clássicos de Brenner e colaboradores em modelos animais, (BRENNER; LAWLER; MACKENZIE, 1996) encontra analogia em situações de excesso de peso, podendo inclusive ser intensificado pelo ambiente inflamatório e metabólico da obesidade (NAUMNIK; MYSLIWIEC, 2010; SILVA JUNIOR et. al., 2017).

\section{Mecanismos inflamatórios e metabólicos}

O tecido adiposo é uma estrutura metabolicamente ativa que tem função importante na regulação do balanço energético. Existem diferentes tipos de tecido adiposo, destacando-se os tecidos adiposos branco, marrom e bege. O tecido adiposo branco, presente em maior quantidade, tem como principal função o estoque de energia por meio do acúmulo de gordura. O tecido adiposo marrom, por sua vez, tem como principal função a dissipação de energia em calor. O tecido adiposo bege desempenha função intermediária, sendo capaz de se transformar em tecido branco e acumular energia, ou se transformar em tecido marrom e dissipar energia sob a forma de calor. Além de funções fisiológicas distintas, estes tipos de tecido adiposo distribuem-se de maneira desigual, do ponto de vista anatômico, existindo predomínio do acúmulo de tecido adiposo branco na região visceral. Esta caracterização é importante, pois, apenas a gordura visceral está implicada nas alterações metabólicas prejudiciais da obesidade (ZHU; SCHERER, 2018).

As diversas funções do tecido adiposo são exercidas através de mediadores que atuam localmente (ação parácrina) e à distância (ação endócrina), denominados adipocinas. Atualmente já estão descritas mais de 600 proteínas secretadas pelo tecido adiposo. Em conjunto, estas substâncias atuam contribuindo para a regulação do apetite e da saciedade, distribuição de gordura corporal, secreção e sensibilidade à insulina, gasto energético, função endotelial, inflamação, PA e homeostase. Quando produzidas em excesso ou de maneira errática, tais substâncias geram alterações metabólicas e inflamatórias que têm papel fundamental na fisiopatologia da obesidade (ZHU; SCHERER, 2018). Em concordância, estudo observacional finlandês que comparou gêmeos univitelinos com diferença de IMC, mostrou que os obesos apresentavam elevação de TNFalfa, CD38, leptina, e redução de adiponectina, quando 
comparados a seus pares não obesos (PIETILÄINEN et al., 2006). Outros estudos demonstraram que a obesidade está associada à elevação de interleucina 6 e 1-alfa, resistina e fator inibidor da migração de macrófagos, todos associados a ações inflamatórias, pró-trombóticas e promotoras de resistência à insulina (TRAYHURN, 2001; FAIN, 2006). Recentemente, estudo transversal brasileiro demonstrou que adolescentes com excesso de peso apresentam níveis elevados de Syndecan-1, associado com disfunção renal e endotelial, o que, no futuro, pode constituir um marcador precoce de evolução para DRC em indivíduos obesos (SABOIA et. al., 2018).

Dentre as alterações metabólicas da obesidade, merece destaque a presença de resistência à insulina, evidenciada pela redução da utilização da glicose pelas células, o que provoca hiperinsulinemia compensatória. Este mecanismo é decorrente do bloqueio das vias intracelulares que levam à exteriorização dos receptores GLUT-4 que captam a glicose em tecidos periféricos sensíveis a ação da insulina (WHALEY-CONNELL; SOWERS, 2017). Em alguns destes tecidos, como rins e vasos sanguíneos, estas mesmas vias intracelulares estão implicadas na ativação da oxido nítrico sintetase endotelial (eNOS), que promove a produção de óxido nítrico (NO), responsável pela vasodilatação. Em casos de resistência à insulina, a eNOS é menos ativada, o que provoca menor disponibilidade de NO, gerando vasoconstrição. Nos rins esse efeito vasoconstritor predomina na arteríola eferente, o que contribui para a hiperfiltração glomerular (JUNCOS; ITO, 1985; MUNIYAPPA; SOWERS, 2012).

A insulina promove também expansão mesangial e fibrose renal. Esse processo é decorrente da ativação de duas vias distintas: a primeira, por meio do fator de crescimento semelhante à insulina 1 (IGF-1) e a segunda, por meio do fator de crescimento tumoral $\beta 1$ (TGF $\beta 1$ ). Enquanto o IGF-1 reduz a atividade da enzima metaloproteinase-2, responsável pela degradação da matriz extracelular, o TGF $\beta 1$ aumenta a expressão do gene do colágeno tipo IV nas células do epitélio tubular. O resultado final é a hipertrofia e a fibrose renal (SARAFIDIS; RUILOPES, 2006; ABRASS; SPICER; RAUGI, 1994; GULER et al., 1989; BAUMANN; EISENHAUER; HARTMANN, 1992). Por fim, a insulina está envolvida na ampliação do efeito vasoconstritor da angiotensina II sobre as arteríolas renais e no aumento da produção de endotelina-1 (REYNOLDS et al., 2016). Como resultado, ocorre proliferação da matriz mesangial, retenção de sódio e vasoconstrição das arteríolas renais (ZICHA et al., 2018).

Outra via que se expressa de maneira patológica no obeso é a da leptina. Secretada predominantemente pelos adipócitos, essa molécula, conhecida como hormônio da saciedade, exerce ações em órgãos periféricos como rins e sistema imune, agindo também no sistema nervoso central, em nível do hipotálamo, promovendo a saciedade e reduzindo a ingestão alimentar. Este hormônio também é responsável por aumento do gasto energético através do estímulo do sistema nervoso simpático, ocasionando aumento da termogênese. Além disso, a interação da leptina com os peroxisome proliferators activador receptors (PPARs), especialmente o PPAR- $\gamma$, contribui para a redistribuição de gordura para o tecido adiposo subcutâneo e reduz o acúmulo da mesma na região central do abdome e em órgãos não adiposos como fígado e rins (UNGER 2002). Contudo, em estudos experimentais com modelos animais, Mark et. al. evidenciaram que animais obesos apresentam dissociação dos efeitos simpaticotônicos e anoréticos da leptina, de tal modo que eles seriam sensíveis à estimulação do SNS, tornando-se hipertensos; mas seriam resistentes aos efeitos anoréticos com diminuição da saciedade o que contribuiria para a deposição central de gordura (MARK et al., 2002).

O acúmulo central de gordura se associa ao fenômeno da lipotoxicidade, que corresponde ao acúmulo anormal de gordura em tecidos não adiposos, principalmente sob a forma de ácidos graxos livres poli-insaturados e seus metabólitos (diacilglicerol e ceramidas). Nos rins, principais órgãos responsáveis pela depuração da leptina, este acúmulo causa estresse oxidativo e resistência à insulina, alterando as vias de sinalização intracelular, e promovendo inflamação, hipertrofia celular, expansão da matriz mesangial, apoptose, fibrose renal e disfunção endotelial (BRIFFA, et al., 2013; IZQUIERDO-LAHUERTA; MARTÍNEZ-GARCIA; MEDINA-GÓMEZ, 2016; UNGER, 2002; VIRTUE, VIDALPUIG, 2010). Desta forma, a hiperleptinemia leva à piora da função renal, que, por sua vez, se relaciona à redução de sua depuração e piora da hiperleptinemia (SHANKAR et al., 2012). Em decorrência destes efeitos, recentemente a leptina tem sido reconhecida como uma importante toxina urêmica (ZHU; SCHERER, 2018).

Em contraposição aos efeitos prejudiciais da insulina e da leptina, o tecido adiposo produz outra substância com efeitos anti-inflamatório e anti-obesidade que aumenta à sensibilidade à insulina: a adiponectina. Esta proteína, presente em três isoformas: de baixo, médio e alto peso molecular, é secretada predominantemente pelo tecido adiposo. Estudos experimentais demonstram que, em células mesangiais cultivadas com adiponectina, ocorre atenuação dos efeitos pro-fibróticos da angiotensina II (GUO et al., 2014). Estes mesmos estudos demonstram que a administração de adiponectina recombinante reduz proteinúria, fibrose renal e marcadores inflamatórios em modelos animais. Outros estudos sugerem ainda que, em modelos animais transgênicos com nefropatia diabética, a super-expressão de adiponectina conduz a aumento dos níveis de nefrina, redução de proteinúria 
e redução da disfunção endotelial (NAKAMAKI et al., 2011). A adiponectina também pode desempenhar efeito nefroprotetor através da redução dos níveis de ceramidas. Tais moléculas são esfingolípides gerados através de ácidos graxos livres e que estão intimamente relacionadas com a lipotoxicidade, por agir na regulação de processos inflamatórios e apoptóticos (MITSNEFES et al., 2014; SAS et al., 2015).

Embora os níveis de adiponectina se encontrem reduzidos na maioria das doenças metabólicas, em condições que cursam com proteinúria e/ou redução da TFG, observa-se aumento dos mesmos, proporcional ao grau de resistência à sua ação (ZHU; SCHERER, 2018). A adiponectina de alto peso molecular é a isoforma que possuiu maior atividade biológica e é predominantemente metabolizada pelo fígado (HALBERG et al., 2009). A ausência de depuração renal levanta a hipótese de que o aumento dos níveis plasmáticos de adiponectina na DRC seja consequência do aumento compensatório da produção, ao invés da redução de sua eliminação. Contudo, adiponectina de baixo peso molecular pode ser encontrada na urina e tem sido implicada na progressão de DRC, em pacientes com nefropatia diabética e também como possível biomarcador de flare renal do lupus eritemaotoso sistêmico (ROVIN et al., 2005; VON EYNATTEN et al., 2009; PANDURU et al., 2015).

Conclui-se, portanto, que as alterações da hemodinâmica glomerular provocam modificações funcionais nos glomérulos, caracterizadas por vasodilatação pré-glomerular, HAS, hiperfiltração glomerular, aumento da reabsorção de sódio e maior secreção de renina. Estas modificações funcionais são intensificadas pelo grau e tempo de instalação da obesidade e pelo ambiente pró-inflamatório e pró-fibrótico típico da obesidade. O resultado final são alterações morfo-estruturais glomerulares, principalmente GESF, que podem se manifestar sob a forma de glomerulopatia associada à obesidade (GAO) (KAMBHAM et al., 2001) bem como pelo agravamento de outras glomerulopatias pré-existentes.

\section{Consequências renais da obesidade}

Os mecanismos supracitados abordam a relação causal entre obesidade e lesão renal, ou seja, obesidade como causa de DRC de novo, também conhecida como $\mathrm{GAO}$, além da piora de possível DRC de base. Esta íntima relação entre obesidade e piora da DRC tem sido demonstrada em estudos com análise multivariada comparando indivíduos obesos e magros acompanhados de maneira prospectiva (FOX et al., 2004; TOZAWA et al., 2002); por estudos que comparam obesos e magros submetidos à nefrectomia para doação de rim (PRAGA et al., 2000) e pela análise da taxa de declínio da função renal residual de pacientes submetidos à diálise peritoneal (HTAY et al., 2016).

Atualmente, a GAO é considerada processo patológico bem reconhecido, caracterizado como uma forma de podocitopatia, em que existe certo grau de hipertrofia glomerular, ou glomerulomegalia, com ou sem GESF e apagamento parcial dos processos podocitários. A GESF, por sua vez, é definida como consolidação segmentar do tufo glomerular, por matriz extracelular e/ou hialina, causando colapso e obliteração glomerular (D'AGATI et al., 2016). Na GAO, a GESF ocorre nos glomérulos hipertróficos, concentrandose próximo ao polo vascular, sendo esta preferência possivelmente relacionada ao feito da hiperfiltração glomerular (D'AGATI et al., 2004). À medida que o glomérulo torna-se hipertrofiado, a densidade do podócito diminui, podendo neste contexto, antecipar o aparecimento da GESF. Em estudo de Fukuda et. al. demostrou-se que o volume glomerular aumenta de forma exponencial ao ganho de peso corporal. De maneira análoga, existe uma tendência ao aumento do volume celular do podócito, embora este incremento não se equipare ao aumento do volume glomerular. 0 resultado final é o desnudamento da membrana basal glomerular em certos pontos, com adesão à cápsula de Bowman e favorecimento ao desenvolvimento de esclerose segmentar (FUKUDA et al., 2012). Estes resultados são parcialmente causados e potencializados pela hiperfiltração glomerular. Desde sua descrição inicial em 1974, a prevalência da GAO aumentou mais de 10 vezes, elevando de 0,2\%, no início da década de 1990 para 2,7\%, em 2015 (D'AGATI et al., 2016).

$\mathrm{Na}$ maioria dos casos de GAO e DRC relacionada à obesidade, há proteinúria subnefrótica. A porcentagem de pacientes com proteinúria nefrótica varia de $10 \%$ a $48 \%$, mas a síndrome nefrótica franca, com hipoalbuminemia, dislipidemia e edema está presente em menos de $10 \%$ dos pacientes, mesmo naqueles com proteinúria massiva (D'AGATI et al., 2016). Esta característica é compartilhada com outros tipos secundários de GESF (PRAGA et al., 1991), o que pode dificultar ou retardar o diagnóstico e o tratamento. Acredita-se que a ausência de síndrome nefrótica franca, mesmo nos casos em que há proteinúria massiva, se deva à instalação lenta e gradual da perda de proteína, o que permite ao fígado criar mecanismos compensatórios, aumentando a produção de albumina e outras proteínas. Outros autores postulam que os mecanismos da proteinúria causada por hiperfiltração glomerular diferem daqueles causados por glomerulopatias primárias (PRAGA; MORALES, 2016).

A compreensão destas particularidades clínicas é importante, pois, pacientes obesos que apresentam proteinúria acompanhada de síndrome nefrótica franca são mais propensos a apresentarem como diagnóstico principal glomerulopatias primárias, tais quais: lesão mínima, GESF primária, glomerulonefrite 
membranosa etc. (PRAGA; MORALES, 2016). Cada uma destas entidades clínicas possui tratamento específico que foge ao objetivo desta breve revisão. Porém, vale ressaltar que a obesidade piora a proteinúria e possivelmente o desfecho das principais glomerulopatias primárias, conforme demonstrado no estudo de Yonekura et. al. Neste estudo, foi revisado e comparado o grau de proteinúria com o peso e a superfície corpórea de mais de 4.000 pacientes do Japan Renal Biopsy Registry (J-RBR), portadores das principais glomerulopatias primárias. Os resultados mostraram que pacientes com lesão mínima, glomerulonefrite membranosa, glomerulonefrite membranoproliferativa e glomerulopatia por IgA associada à obesidade apresentaram maior proteinúria. O mesmo não foi identificado nos pacientes com GESF e glomerulopatia proliferativa mesangial nãoIgA, acreditando-se que estas tenham mecanismos de proteinúria diferentes da hiperfiltração glomerular do obeso (YONEKURA et al., 2014). No mesmo sentido, Bonnet et. al, demonstraram que pacientes com nefropatia por IgA e com IMC acima de $25 \mathrm{~kg} / \mathrm{m}^{2}$ desenvolvem lesões histológicas mais complexas e pior desfecho renal quando comparados a indivíduos magros (BONNET et al., 2001).

Em contraposição a estes achados, fato ainda não totalmente elucidado e que carece de mais estudos, é a chamada epidemiologia reversa da obesidade, também denominada paradoxo da obesidade. Vários estudos observacionais têm demonstrado que indivíduos obesos com DRC terminal, inscritos em programa de terapia renal substitutiva, têm menor mortalidade quando comparados a indivíduos não obesos, principalmente na população de pacientes submetidos à hemodiálise (PARK et al., 2014). Estes resultados também são observados em outras doenças crônicas como doença pulmonar obstrutiva crônica, insuficiência cardíaca congestiva, infecção pelo vírus da imunodeficiência humana e malignidades (RHEE; AHMADI; KALANTAR-ZADEH, 2016).

Várias hipóteses tentam justificar estes achados. Alguns autores postulam que, em parte, o paradoxo da obesidade pode ser explicado pelo fato de que a maioria dos estudos utilizam como medida da obesidade unicamente o IMC, índice que não permite a distinção entre massa magra e gordura, desconsiderando as medidas mais fidedignas ao acúmulo prejudicial de gordura como circunferência abdominal e razão cinturaquadril. Dessa forma, o suposto efeito protetor poderia ser decorrente da maior massa muscular e não do maior acúmulo de gordura. Outros postulam que indivíduos obesos possuem melhor aporte nutricional, resistindo à caquexia causada por condições inflamatórias crônicas, como a DRC. Além disto, dada à elevada mortalidade da doença, os riscos cardiovasculares clássicos relacionados à obesidade e que são responsáveis por maior parte da morbimortalidade do excesso de peso, não tenham tempo de se desenvolver e impactar na mortalidade final do paciente (KALANTAR-ZADEH et al., 2017).
Conclui-se, portanto, que a obesidade seja causa de DRC e fator de pior evolução de DRC previamente estabelecida. Por outro lado, pacientes com DRC em fases avançadas, quando analisados apenas pelo IMC, têm curva de mortalidade em jota, sendo prejudiciais os extremos de IMC. Dessa forma, fato que precisa ser mais bem esclarecido em estudo futuros estudos é se a obesidade, per se, constitui fator protetor na DRC e quais seriam os mecanismos envolvidos neste paradoxo (NADERI et al., 2018).

\section{Tratamento da DRC relacionada à obesidade}

Diante do exposto, conclui-se que o excesso de peso é um importante fator de risco para o desenvolvimento e agravamento da DRC, independente de sua associação com HAS e com DM2. Assim, a prevenção e o tratamento da obesidade representam a principal forma para a prevenção primária e secundária da DRC. Mudança de estilo de vida como a prática regular de exercícios físicos e a reeducação alimentar são medidas eficazes para a redução do risco de desenvolvimento e progressão da DRC, em pacientes obesos e com sobrepeso (WING, et al., 2013). Paralelamente, a perda de peso promove redução da PA, atenua a hiperfiltração glomerular e reduz a proteinúria (BOLIGNANO; ZOCCALI, 2013), ações reconhecidamente nefroprotetoras.

De maneira similar à perda de peso, os bloqueadores dos receptores de angiotensina (BRA) ou os inibidores da enzima conversora de angiotensina (IECA) induzem redução substancial da proteinúria (D'AGATI et al., 2016). Além disso, estudos experimentais com BRA mostram que existe atenuação da fibrose tubulointersticial e da progressão de DRC através da redução da expressão de TGF- $\beta 1$ e do inibidor do ativador do plasminogênio-1 (PAI1) que promovem expansão da matriz mesangial no rim (REDDY, et al., 2014; PANDEY, et al., 2016). Do mesmo modo, o bloqueio dos receptores mineralocorticóides reduz a hiperfiltração e a proteinúria, podendo contribuir para o retardo da progressão da DRC. Contudo, mais estudos são necessários para confirmar a efetividade dessa estratégia na nefroproteção, em indivíduos com excesso de peso (SCHWENK; HIRSCH; BOMBACK, 2015).

\section{CONCLUSÃO}

A obesidade é uma doença de proporções epidêmicas, de mecanismos fisiopatológicos complexos e que cursa com envolvimento multissistêmico (Figura 1). Sua correlação com condições crônicas de saúde, com destaque para a DRC, promove aumento de risco cardiovascular, sendo causa de elevada morbidade e mortalidade. O entendimento destes mecanismos pode permitir a identificação precoce de pacientes de risco, a instituição de medidas preventivas e a abordagem de possíveis danos a órgãos alvo desta população. 


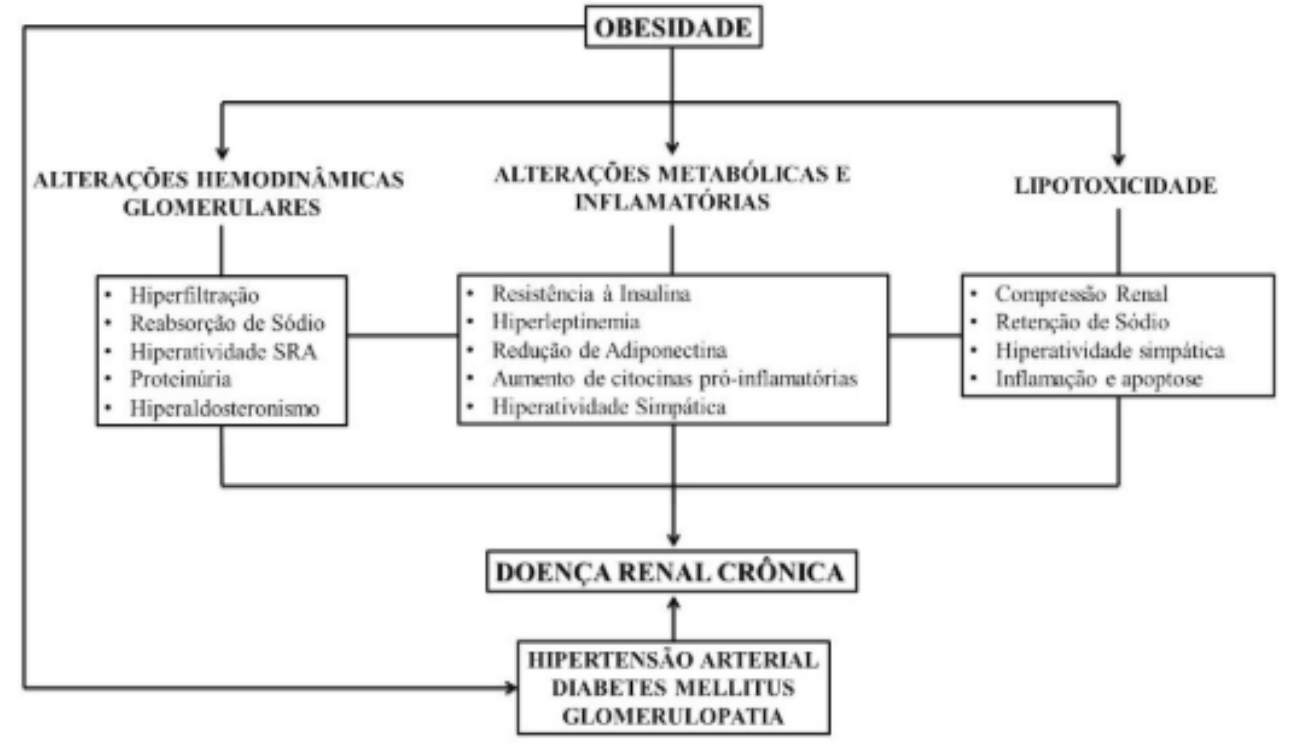

Figura 1: Obesidade como causa de doença renal crônica - Mecanismos: alterações hemodinâmicas, metabólicas e lipotoxidade, frequentemente associadas à hipertensão arterial, diabetes mellitus e glomerulopatia podem determinar DRC. SRA, sistema renina angiotenisa; DRC, doença renal crônica.

\section{REFERÊNCIAS}

ABRASS, C. K; SPICER, D.; RAUGI, G. J. Insulin induces a chance in extracellular matrix glycoproteins synthesized by rat mesangial cells in culture. Kidney International, v. 46, p. 613-620, sep. 1994.

BAUMANN, U.; EISENHAUER, T.; HARTMANN, H. Increase of glomerular filtration rate and renal plasma flow by insulin-like growth factor-1 during euglycaemic clamping in anaesthetized rats. European Journal of Clinical Investigation, v. 22, n.3, p. 204-209, mar. 1992.

BOLIGNANO, D.; ZOCCALI, C. Effects of weight loss on renal function in obese CKD patients: a systematic review. Nephrology Dialysis Transplantation, v.28, s. 4, p. 82-98, nov. 2013.

BONNET, F. et. al. Excessive body weight as a new independent risk factor for clinical and pathological progression in primary IgA nephritis. American Journal of Kidney Diseases, v. 37, n. 4, p. 720-727, apr. 2001.

BRENNER, B. M; LAWLER, E. V.; MACKENZIE, H. S. The hyperfiltration theory: a paradigm shift in nephrology. Kidney International. $v$. 49, n. 6, p. 1774-1777, jun. 1996.

BRIFFA, J. F. et al. Adipokines as a link between obesity and chronic kidney disease. American Journal of Physiology-Renal Physiology, v. 305, n. 12, p. 1629-1636, dec. 2013.

CHAGNAC, A. et al. Glomerular hemodynamics in severe obesity. American Journal of Physiology-Renal Physiology, v. 278, $n$. 5, p. 817-822, may. 2000.
COLE, B. K. et al. Valsartan protects pancreatic islets and adipose tissue from the inflammatory and metabolic consequences of a high-fat diet in mice. Hypertension, v. 55, n. 3, p. 715-721, mar. 2010.

D'AGATI, V. D. et al. Pathologic classification of focal segmental glomerulosclerosis: a working proposal. American Journal of Kidney Diseases, v. 43, n. 2, p. 368-382, feb. 2004.

D'AGATI, V. D.; et al. Obesity-related glomerulopathy: clinical and pathologic characteristics and pathogenesis. Nature Reviews Nephrology, v. 12, n. 8, p. 453-471, aug. 2016.

DE PAULA, R. B.; SILVA, A. A.; HALL, J. E. Aldosterone antagonism attenuates obesity-induced hypertension and glomerular hyperfiltration. Hypertension, v. 43, n. 1, p. 41-47, jan. 2004.

FAIN, J. N. Release of interleukins and other inflammatory cytokines by human adipose tissue is enhanced in obesity and primarily due to the nonfat cells. Vitamins \& Hormones, v. 74, p. 443-477, 2006.

FOX, C. S. Predictors of new-onset kidney disease in a communitybased population. Journal of American Medical Association. v. 291, n. 7, p. 844, feb. 2004.

FRIGOLET, M. E.; TORRES, N.; TOVAR, A. R. The renin-angiotensin system in adipose tissue and its metabolic consequences during obesity. The Journal of Nutritional Biochemistry, v. 24 n. 12, p. 2003-2015, dec. 2013.

FUKUDA, A.; et al. Growth-dependent podocyte failure causes glomerulosclerosis. Journal of the American Society of Nephrology, v. 23, n. 8, p. 1351-1363, aug. 2012.

GULER, H. P.; et al. Insulin-like growth factor-1 increases glomerular filtration rate and renal plasma flow in man. Acta Endocrinology. v. 121, n. 1, p 101-106, jul. 1989. 
GUO, H.; et al. Protective effects of glucagon-like peptide-1 analog on renal tubular injury in mice on high-fat diet. Cellular Physiology and Biochemistry, v. 41, n. 3, p.1113-1124, feb. 2017.

GUO, C.; et al. Mineralocorticoid receptor blockade reverses obesity-related changes in expression of adiponectin, peroxisome proliferator-activated receptor-gamma, and proinflammatory adipokines. Circulation. V. 117, n. 17, p. 2253-2261, apr. 2008.

GUO, X.; et al. Adiponectin retards the progression of diabetic nephropathy in $\mathrm{db} / \mathrm{db}$ mice by counteracting angiotensin II. Physiological Reports, v. 2, n. 2, e. 00230, feb. 2014.

HALBERG, N.; et al. Systemic fate of the adipocyte-derived factor adiponectin. Diabetes, v. 58, n. 9, p. 1961-1970, set. 2009.

HALL, J. E. The kidney, hypertension and obesity. Hypertension. v. 41, n. 3, p. 625-633, jan. 2003.

HALL, J. E.; et al. Obesity-induced hypertension. Renal function and systemic hemodynamics. Hypertension, v. 22, n. 3, p. 292-299, sep. 1993.

HENEGAR, J. R.; et al. Functional and structural changes in the kidney in the early stages of obesity. Journal of American Society of Nephrology. v.12, n. 6, p. 1211-1217, jun. 2001.

HIRATA, A.; et al. Contribution of glucocorticoid-mineralocorticoid receptor pathway on the obesity-related adipocyte dysfunction. Biochemical and Biophysical Research Communications. v. 419, n. 12, p. 182-187, mar. 2012.

HTAY, H.; et al. Predictors of residual renal function decline in peritoneal dialysis patients: the bal ANZ trial. Peritoneal Dialysis International, v. 37 n. 3, p. 283-289, may./jun. 2016.

IZQUIERDO-LAHUERTA， A.; MARTÍNEZ-GARCÍA， C.; MEDINAGÓMEZ, G. Lipotoxicity as a trigger factor of renal disease. Journal of Nephrology, v. 29, n. 5, p. 603-610, oct. 2016.

KALANTAR-ZADEH, K.; et. al. The obesity paradox in kidney disease: how to reconcile it with obesity management. Kidney International Reports, v. 2, n. 2, p. 271-281, mar. 2017.

KAMBHAM, N.; et al. Obesity-related glomerulopathy: an emerging epidemic. Kidney International, v. 59, n. 4, p. 1498-1509, abr. 2001.

LEMARIÉ, C.A.; SCHIFFRIN, E. L. The angiotensin II type 2 receptor in cardiovascular disease. Journal of Renin Angiotensin Aldosterone System, v. 11, n. 1, p. 19-31, mar. 2010.

MA, L.; et al. Angiotensin type 1 receptor modulates macrophage polarization and renal injury in obesity. American Journal of Physiology-Renal Physiology, v. 300, n. 5, p. 1203-1213, may. 2011.

MARK, A. L.; et al. Selective leptin resistance: a new concept in leptin physiology with cardiovascular implications. Journal of Hypertension, v. 20, n. 7, p. 1245-1250, jun. 2002.
MASSIERA, F. et al. Adipose angiotensinogen is involved in adipose tissue growth and blood pressure regulation. The FASEB Journal, v. 15, n. 14 , p. $2727-2729$, dec. 2001.

MINISTÉRIO DA SAÚDE. Vigitel Brasil 2016: Vigilância de fatores de risco e proteção para doenças crônicas por inquérito telefônico. Ministério da Saúde, Secretaria de Vigilância em saúde, Secretaria de Gestão Estratégica e Participativa. Brasília, 2016. Disponível em: http://portalms.saude.gov.br/images/pdf/2018/marco/02/vigitelbrasil-2016.pdf. E http://portalarquivos.saude.gov.br/images/ pdf/2017/abril/17/Vigitel.pdf. Acesso em 28/09/2018, 17:43.

MIRANDA, L. S. P.; DE PAULA, R.B.; COSTA, M. B. Fatores dietéticos de risco e de proteção para condições crônicas de saúde em município da zona da mata de Minas Gerais. 113f. Dissertação (Mestrado em Saúde Brasileira). Programa de pós-graduação da Universidade Federal de Juiz de Fora, Juiz de Fora, 2017.

MITSNEFES, M. et al. Ceramides and cardiac function in children with chronic kidney disease. Pediatric Nephrology, v. 29, n. 3, p. 415-422, mar. 2014.

MUNIYAPPA, R.; SOWERS, J. R. Endothelial insulin and IGF-1 receptors: when yes means no. Diabetes, v. 61, n. 9, p. 2225-2227, sep. 2012.

NADERI, N. et al. Obesity paradox in advanced kidney disease: from bedside to the bench. Progress in Cardiovascular Diseases, $v$. 61, n. 2, p. 168-181, jul./ago. 2018.

NAKAMAKI, S. et al. Adiponectin reduces proteinuria in streptozotocin-induced diabetic Wistar rats. Experimental Biology and Medicine, v. 236, n. 5, p. 614-620, may. 2011.

NAUMNIK, B.; MYSLIWIEC, M. Renal consequences of obesity. Medical Science Monitor. V. 16, n.8, p. 163-170, aug. 2010.

NGUYEN, A.; CAT, D.; TOUYZ, R.M. A new look at the reninangiotensin system - focusing on the vascular system. Peptides, v. 32 , n. 10 , p. $2141-2150$, oct. 2011.

PANDEY, A. et al. H2AK119 monoubiquitination regulates angiotensin II receptor mediated macrophage infiltration and renal fibrosis in type 2 diabetic rats. Biochimie, v. 131, p. 68-76, dec. 2016.

PANDURU, N. M. et al. Urinary adiponectin is an independent predictor of progression to end-stage renal disease in patients with type 1 diabetes and diabetic nephropathy. Diabetes Care, v. 38, n. 5, p. 883-890, may. 2015.

PARK, J. et al. Obesity Paradox in End-Stage Kidney Disease Patients. Progress in Cardiovascular Diseases, v. 56, n. 4, p. 415-425, jan./fev. 2014.

PIETILÄINEN, K. H. et al. Acquired obesity increases CD68 and tumor necrosis factor- $a$ and decreases adiponectin gene expression in adipose tissue: A study in monozygotic twins. The Journal of Clinical Endocrinology \& Metabolism, v. 91, n. 7, p. 2776-2781, jul. 2006. 
PRAGA, M. et al. Nephrotic proteinuria without hypoalbuminemia: clinical characteristics and response to angiotensin-converting enzyme inhibition. American Journal of Kidney Diseases, v. 17, n. 3, p. 330-338, mar. 1991.

PRAGA, M. et al. Influence of obesity on the appearance of proteinuria and renal insufficiency after unilateral nephrectomy. Kidney International, v. 58, n. 5, p. 2111-2118, nov. 2000.

PRAGA, M.; MORALES, E. The fatty kidney: obesity and renal disease. Nephron, v. 136, n. 4, p. 273-276, jul. 2016.

REDDY, M. A. et al. Losartan reverses permissive epigenetic changes in renal glomeruli of diabetic $\mathrm{db} / \mathrm{db}$ mice. Kidney International, $\mathrm{v}$. 85, n. 2, p. 362-373, fev. 2014.

REMUZZI, G.; CATTANEO, D.; PERICO, N. The aggravating mechanisms of aldosterone on kidney fibrosis. Journal of the American Society of Nephrology, n. 19, v. 8, p. 1459-1462, aug. 2008

REYNOLDS, L. J., et al. Obesity, type 2 diabetes, and impaired insulin-stimulated blood flow: role of skeletal muscle NO synthase and endothelin-1. Journal of Applied Physiology, v. 122, n. 1, p. 38-47, jan. 2017.

RHEE, C. M.; AHMADI, S. F.; KALANTAR-ZADEH, K. The dual roles of obesity in chronic kidney disease. Current Opinion in Nephrology and Hypertension, v. 25, n. 3, p. 208-216, may. 2016.

ROVIN, B. H.; et al. Plasma, urine, and renal expression of adiponectin in human systemic lupus erythematosus. Kidney International, v. 68, n. 4, p. 1825-1833, oct. 2005.

SABOIA, Z. M. R. M. et al. Association between syndecan-1 and renal function in adolescents with excess weight: evidence of subclinical kidney disease and endothelial dysfunction. Brazilian Journal of Medical Biology and Research. v. 51, n. 3, p. 71-71, jan. 2018.

SARAFIDIS, P. A.; RUILOPE, L. M. Insulin resistance, hyperinsulinemia, and renal injury: mechanisms and implications. American Journal of Nephrology, v. 26, n. ?, p. 232-244, may. 2006.

SAS, K. M. et al. Targeted lipidomic and transcriptomic analysis identifies dysregulated renal ceramide metabolism in a mouse model of diabetic kidney disease. Journal of Proteomics \& Bioinformatics, s. 14:002, may. 2015.

SCHIMIDT, M. I.; et al. Cohort Profile: Longitudinal Study of Adult Health (ELSA-Brasil). International Journal of Epidemiology. v. 44 , n. 1, p. 68-75, feb. 2015

SCHWENK, M. H.; HIRSCH, J. S.; BOMBACK, A. S. Aldosterone blockade in CKD: emphasis on pharmacology. Advances in Chronic Kidney Disease, v. 22, n. 2, p. 123-132, mar. 2005.
SILVA JUNIOR, et al. Obesity and kidney disease. Jornal Brasileiro de Nefrologia. v. 39, n. 1, p. 65-69, mar. 2017.

TIROSH, A.; GARG, R.; ADLER, G. K. Mineralocorticoid receptor antagonists and the metabolic syndrome. Current Hypertension Reports. v. 12, n. 4, p. 252-257, aug. 2010.

TOZAWA, M. et al. Influence of smoking and obesity on the development of proteinuria. Kidney International, v. 62, n. 3, p. 956-962, sep. 2002.

TRAYHURN, P.; BEATTIE, J. H. Physiological role of adipose tissue: white adipose tissue as an endocrine and secretory organ. Proceedings of the Nutrition Society, v. 60, n. 03, p. 329-339, aug. 2001.

UNGER, R. H. Lipotoxic diseases. Annual Review of Medicine, v. 53, n. 1, p. 319-336, feb. 2002.

VIRTUE, S.; VIDAL-PUIG, A. Adipose tissue expandability, lipotoxicity and the metabolic syndrome - an allostatic perspective. Biochimica et Biophysica Acta (BBA) - Molecular and Cell Biology of Lipids, v. 1801, n. 3, p. 338-349, mar. 2010.

VON EYNATTEN, M. et al. (2009). Urinary adiponectin excretion: a novel marker for vascular damage in type 2 diabetes. Diabetes, $v$. 58, n. 9, p. 2093-2099, sep. 2009.

WHALEY-CONNELL, A.; SOWERS, J. R. Obesity and kidney disease: from population to basic science and the search for new therapeutic targets. Kidney International, v. 92, n. 2, p. 313-323, aug. 2017

WING, R. et al. Cardiovascular effects of intensive lifestyle intervention in type 2 diabetes. New England Journal of Medicine, v. 369, n. 2, p. 145-154, jul. 2013.

YIANNIKOURIS, F. et al. Adipocyte-specific deficiency of angiotensinogen decreases plasma angiotensinogen concentration and systolic blood pressure in mice. American Journal of Physiology-Regulatory, Integrative and Comparative Physiology. v. 302, n. 2, p. 244-251, jan. 2012.

YONEKURA, Y. et al. The influences of larger physical constitutions including obesity on the amount of urine protein excretion in primary glomerulonephritis: research of the Japan Renal Biopsy Registry. Clinical and Experimental Nephrology, v. 19, n. 3, p. 359-370, jun. 2014.

ZHU, Q.; SCHERER, P. E. Immunologic and endocrine functions of adipose tissue: implications for kidney disease. Nature Reviews Nephrology, v. 14, n. 2, p. 105-120, feb. 2018.

ZICHA, J. et al. Renoprotective effects of ETA receptor antagonists therapy in experimental non-diabetic chronic kidney disease: is there still hope for the future?. Physiological Research, v. 67, s. 1, p. 55-67, jun. 2018. 